\title{
Van der Waals-Maxwell Theory, Lebowitz-Penrose Limit and Superstable Interactions
}

\author{
A. Gerardi, C. Marchioro * and E. Olivieri * \\ Istituto Matematico, Università di Roma, Roma, Italia \\ E. Presutti \\ Istituto di Fisica, Università dell'Aquila, L'Aquila, Italia
}

Received July 27, 1972

\begin{abstract}
We obtain rigorous results about the liquid-vapour phase transition. We prove that in the Lebowitz-Penrose limit the hypothesis of hard core for the interaction is not essential to reproduce the Van der Waals-Maxwell theory. It can be replaced by the superstability.
\end{abstract}

\section{Introduction}

Many efforts have been devoted to the deduction of the features of liquid-vapor phase transition from the fundamental principles of statistical mechanics. Some authors have treated the problem by introducing a long range interaction [1-4]. In particular Lebowitz-Penrose (henceforth referred to as LP) have considered a system of classical particles interacting pairwise in $v$ dimensions via a potential of the form

$$
v(r, \gamma)=q(r)+\gamma^{v} \varphi(\gamma r)
$$

where $r$ is the vector distance between a pair of particles, and $\gamma$ is a positive parameter; $q(r)$ is the "reference potential": it has a hard core and decreases in an integrable way to infinity; $\gamma^{v} \varphi(\gamma r)$ is the "Kac potential": $\gamma$ fixes simoultaneously the range and the strength.

LP have studied the thermodynamic functions in the limit $\gamma \rightarrow 0$. Namely they first carried out the thermodynamic limit and then considered the range of the Kac potential increasing to infinity. The most interesting Kac potentials from the physical point of view are the nonrepulsive one, i.e. $\varphi \leqq 0$; in this case LP have shown that

$$
a\left(\varrho, 0^{+}\right)=\lim _{\gamma \rightarrow 0} a(\varrho, \gamma)=\operatorname{CE}\left(a^{0}(\varrho)+\frac{1}{2} \alpha \varrho^{2}\right)
$$

$\star$ Research partially supported by the Consiglio Nazionale delle Ricerche (G.N.F.M.A.M.F.I.). 
where $\varrho$ is the density, $a(\varrho, \gamma)$ is the free energy density, $a^{0}(\varrho, \gamma)$ is the free energy density for the reference system (that is for $\varphi=0$ ), $\alpha=\int_{\mathbb{R}^{v}} \varphi(r) d r$; $C E f(\varrho)$ means the convex envelope of $f(\varrho)$, i.e. the maximal convex function not greater than $f(\varrho)$.

LP have also shown the equivalence of Eq. (1.2) with the Van der Waals-Maxwell equal area rule [5] applied to

$$
\pi(\varrho)=\pi_{0}(\varrho)+\frac{1}{2} \alpha \varrho^{2}
$$

where $\pi_{0}(\varrho)$ is the pressure of the reference system. Eq. (1.3) is analogous to the Van der Waals equation of state.

On the same problem Gates and Penrose [4] have obtained more general results; in particular they carried out an extensive discussion of the properties of the Kac potential necessary to produce the Maxwell rule.

In all these papers it has been supposed the presence of a hard core in the reference system. This is needed to exclude a too high local density and so bound the interaction between different regions of the system. On the other hand the conjecture has been made that such a restrictive hypothesis is only technical and unessential to explain the condensation [2].

In this paper we deduce Eq. (1.2) assuming only the superstability of the interaction potential ${ }^{1}$. In fact superstability avoids large fluctuations of the local density [6]. In this paper we show that it is possible to give probability estimates of the large densities which are uniform in the range of the Kac potential, $\gamma$, and this allows us to achieve the desired result.

In the spirit of the present work, whose aim is to prove this generalization, we confine ourselves to the physical interesting case of non repulsive Kac potentials.

\section{Statements and Results}

We consider a system of identical particles interacting pairwise via a potential $v_{\gamma}$. We make the following assumptions.

D.1. Two body interaction. Let $q: \mathbb{R}^{v} \rightarrow \mathbb{R} \cup \infty$ and $\varphi: \mathbb{R}^{v} \rightarrow \mathbb{R}^{-}$then for $\gamma \in(0,1]$ we define

$$
v_{\gamma}(x)=q(x)+\gamma^{v} \varphi(x) \quad x \in \mathbb{R}^{v}
$$

$\varphi(x)$ is supposed Riemann integrable in the whole space. We put

$$
\alpha=\int_{\mathbb{R}^{v}} \varphi(x) d x .
$$

${ }^{1}$ As stressed by Ruelle [6] this is not a real loss of generality with respect to the class of stable interactions.

${ }^{2} \mathbb{R}^{+}\left[\mathbb{R}^{-}\right]$denotes real non negative [non positive] numbers. 
D.2. Potential energy. Let $\Lambda \subset \mathbb{R}^{v}$ be Lebesgue measurable. For $s \in \mathbb{Z}^{+}$, $x_{1}, \ldots, x_{s} \in \Lambda$ we define

$$
\begin{aligned}
U\left(x_{1}, \ldots, x_{s}\right) & =\sum_{i<j}\left\{q\left(x_{i}-x_{j}\right)+\gamma^{v} \varphi\left[\gamma\left(x_{i}-x_{j}\right)\right]\right\}, \\
U^{0}\left(x_{1}, \ldots, x_{s}\right) & =\sum_{i<j} q\left(x_{i}-x_{j}\right) .
\end{aligned}
$$

D.3. Let $0<\lambda \in \mathbb{R}$. We define

$$
\Gamma_{l}(r)=\left\{x \in \mathbb{R}^{v}:\left(r^{i}-\frac{1}{2}\right) \lambda l \leqq x^{i}<\left(r^{i}+\frac{1}{2}\right) \lambda l \quad i=1, \ldots, v\right\} \quad 0<l \in \mathbb{Z}^{+} .
$$

In the sequel we will use the notations

$$
\begin{gathered}
|x|=\max _{1 \leqq i \leqq \nu} x^{i} \quad x \in \mathbb{R}^{v} \\
\mathscr{I}(a) \text { integer part of } a, \quad a \in \mathbb{R}^{+} .
\end{gathered}
$$

D.4. Lower regularity. Let $Q: \mathbb{Z}^{+} \rightarrow \mathbb{R}^{+}, \Phi: \mathbb{R}^{+} \rightarrow \mathbb{R}^{+}$satisfy

(a) $Q$ and $\Phi$ are decreasing and $\Phi$ is continuous.

(b) $\inf _{\substack{x \in \Gamma_{1}(r) \\ y \in \Gamma_{1}(0)}} q(x-y) \geqq-Q(|r|)$. $y \in \Gamma_{1}(0)$

(b') $\inf _{\substack{x \in \Gamma_{1}(r) \\ \gamma \in \Gamma_{1}(0)}} \gamma^{v} \varphi[\gamma(x-y)] \geqq-\gamma^{v} \Phi[\gamma|r|]$.

(c) $\sum_{r \in \mathbb{Z}^{\nu}} Q(|r|)<\infty$.

(c) $\sum_{r \in \mathbb{Z}^{v}} \Phi(|r|)<\infty$.

Note that since $\Gamma_{1}(r)$ depends on $\lambda$ the same happens for $Q$ and $\Phi$.

Lemma 2.1. $Q$ and $\Phi$ satisfy D.4. Then the following holds

(i) $\gamma^{v} \sum_{r \in \mathbb{Z}^{v}} \Phi(\gamma|r|)=\hat{\Phi}_{\gamma}<\infty \quad$ for $\quad \gamma \in(0,1]$.

(ii) $\sup _{\gamma \in(0,1]} \hat{\Phi}_{\gamma}=\hat{\Phi}<\infty$.

(iii) Define for $\gamma \in(0,1]$ the family of functions $\left\{\psi_{\gamma}(x)\right\}$ as follows: for $x \in \Gamma_{\gamma}(s) \psi_{\gamma}(x)=\Phi(\gamma|s|)$. Then

$$
\lim _{\gamma \rightarrow 0} \psi_{\gamma}(x)=\Phi(|x|) \quad x \in \mathbb{R}^{v} .
$$

Further there exists a measurable function $\psi(x)$ such that $\psi(x) \geqq \psi_{\gamma}(x)$, $\int_{\mathbb{R}^{v}} d x \psi(x)<\infty$.

(iv) $\lim _{\gamma \rightarrow 0} \hat{\Phi}_{\gamma}=\lambda^{-v} \int_{\mathbb{R}^{v}} d x \Phi(|x|) \equiv \hat{\Phi}_{0}$.

Proof. (i) Since $\Phi(|x|)$ is decreasing, we have

$$
\hat{\Phi}_{\gamma} \leqq \mathscr{I}\left(\gamma^{-v}+1\right) \gamma^{\nu} \sum_{r \in \mathbb{Z}^{\nu}} \Phi(|r|)<2 \sum_{r \in \mathbb{Z}^{\nu}} \Phi(|r|)<\infty .
$$


(ii) The above inequality proves also statement (ii).

(iii) The continuity of $\Phi(|x|)$ proves Eq. (2.6). Then define $\psi(x)$ as follows for

$$
|x|<1 \quad \psi(x)=\Phi(|0|), \quad \text { for } \quad|x| \geqq 1 \quad \psi(x)=\Phi(|x|-1) .
$$

Since $\Phi$ is decreasing $\psi(x) \geqq \psi_{\gamma}(x)$ for $\gamma \in(0,1]$ and

$$
\begin{aligned}
\int_{\mathbb{R}^{v}} d x \psi(x) \leqq \Phi(|0|) & +\sum_{0 \neq r \in \mathbb{Z}^{v}} \Phi(|r|-1) \leqq \Phi(|0|) \\
& +\sum_{l=0}^{\infty}\left[(2 l+3)^{v}-(2 l+1)^{v}\right] \Phi(l)<+\infty .
\end{aligned}
$$

(iv) We obviously have

$$
\int_{\mathbb{R}^{v}} d x \psi_{\gamma}(x)=\lambda^{v} \gamma^{v} \sum_{r \in \mathbb{Z}^{v}} \Phi(\gamma|r|)
$$

then applying Lebesgue theorem through statement (iii) the thesis is completed.

Q.E.D.

D.5. Superstability. There exist $A^{0}>\hat{\Phi}, B \geqq 0$ such that, if $\mathscr{R}$ is a finite subset of $\mathbb{Z}^{v}$ and

$$
\begin{gathered}
X=\left(x_{1}, \ldots, x_{m}\right) \subset \bigcup_{r \in \mathscr{R}} \Gamma_{1}(r), \\
U^{0}\left(x_{1}, \ldots, x_{m}\right) \geqq \sum_{r \in \mathscr{R}}\left[A^{0} n^{2}(X, r)-B n(X, r)\right], \\
n(X, r)=\operatorname{Card}\left(X \cap \Gamma_{1}(r)\right) .
\end{gathered}
$$

We remark that $A^{0}$ and $B$ depend on the choice of $\lambda$.

Note. The condition $A^{0}>\hat{\Phi}$ is too strong for our purposes since we are not really interested in studying the problem in the whole range $\gamma \in(0,1]$ for the Kac potential, while $\hat{\Phi}$ defined as a supremum of $\hat{\Phi}_{\gamma}$ for $\gamma \in(0,1]$ will depend on all the values of $\gamma$. The condition that really matters is that $A^{0}>\hat{\Phi}_{0}$, but from statement (iv), Lemma 2.1, there exists then $0<\gamma_{0}<1$ such that $A^{0}>\Phi_{\gamma_{0}}$. Considering then D.1, ., D.5 defined for $\gamma \in\left(0, \gamma_{0}\right]$, we are reconducted to the previous case and if we read $\varphi\left(\gamma_{0} x\right)$ instead of $\varphi(x)$ we can again consider $\gamma \in(0,1]$.

Lemma 2.2. Let D.1, ., D. 5 hold, then there exists $A>0$ such that for $\mathscr{R}$ a finite subset of $\mathbb{Z}^{v}$ and for every $X=\left(x_{1}, \ldots, x_{m}\right) \subset \bigcup_{r \in \mathscr{R}} \Gamma_{1}(r)$ the
following is true;

$$
U\left(x_{1}, \ldots, x_{m}\right) \geqq \sum_{r \in \mathscr{R}}\left[A n^{2}(X, r)-B n(X, r)\right] .
$$


Proof. We have

$$
\begin{aligned}
U\left(x_{1}, \ldots, x_{m}\right)= & U^{0}\left(x_{1}, \ldots, x_{m}\right)+\gamma^{v} \sum_{i<j}^{1, \ldots, m} \varphi\left[\gamma\left(x_{i}-x_{j}\right)\right] \\
\geqq & \sum_{r \in \mathscr{R}}\left[A^{0} n^{2}(X, r)-B n(X, r)\right] \\
& -\gamma^{v} \sum_{r \in \mathscr{R}} n(X, r) n(X, s) \Phi(\gamma|r-s|) \\
\geqq & \sum_{r \in \mathscr{R}}\left\{A^{0} n^{2}(X, r)-B n(X, r)-\gamma^{v} n^{2}(X, r) \sum_{s \in \mathscr{R}} \Phi(\gamma|r-s|)\right\} \\
\geqq & \sum_{r \in \mathscr{R}}\left\{\left[A^{0}-\hat{\Phi}\right] n^{2}(X, r)-B n(X, r)\right\} .
\end{aligned}
$$

The Lemma is then proved with $A=A^{0}-\hat{\Phi}>0$.

Q.E.D.

We remark that Lemma 2.2 implies " $\gamma$ uniform" superstability of $U$. This follows from the hypothesis D.5, namely that $A^{0}>\hat{\Phi}$.

D.6. Weak tempering. There exist $k \geqq 0, \alpha>v, x_{0}>0$ so that

$$
\begin{aligned}
& q(x) \leqq k|x|^{-\alpha}, \quad|x|>x_{0} . \\
& \text { D.7. } Z(\mu, \Lambda, \gamma)=\sum_{n=0}^{\infty} e^{\beta \mu n} Z_{c}(n, \Lambda, \gamma) \text {, } \\
& Z_{c}(n, \Lambda, \gamma)=\frac{\bar{\lambda}^{n}}{n !} \int_{\Lambda^{n}} d x_{1} \ldots d x_{n} \exp \left[-\beta U\left(x_{1} \ldots x_{n}\right)\right], \\
& Z^{0}(\mu, \Lambda)=\sum_{n=0}^{\infty} e^{\beta \mu n} Z_{c}^{0}(n, \Lambda), \\
& Z_{c}^{0}(n, \Lambda)=\frac{\bar{\lambda}^{n}}{n !} \int_{\Lambda^{n}} d x_{1} \ldots d x_{n} \exp \left[-\beta U^{0}\left(x_{1} \ldots x_{n}\right)\right], \\
& a_{c}=-\frac{1}{\beta|\Lambda|} \ln Z_{c}(n, \Lambda, \gamma), \quad a_{c}^{0}=-\frac{1}{\beta|\Lambda|} \ln Z_{c}^{0}(n, \Lambda),
\end{aligned}
$$

where $\bar{\lambda} e^{\beta \mu}$ is the activity.

Assuming D.4, D.5 and D.6 the thermodynamic limit exists for increasing regions in the Fisher sense [7].

$$
\begin{aligned}
& P(\mu, \gamma)=\lim _{|\Lambda| \rightarrow \infty} \frac{1}{\beta|\Lambda|} \ln Z(\mu, \Lambda, \gamma), \\
& a(\varrho, \gamma)=\lim _{\substack{|\Lambda| \rightarrow \infty \\
\frac{N}{|\Lambda|} \rightarrow \varrho}}-\frac{1}{\beta|\Lambda|} \ln Z_{c}(N, \Lambda, \gamma) .
\end{aligned}
$$

$P^{0}, a^{0}$ are analogously defined for the reference system. 
In the sequel we shall need an estimate on the probability of local high densities. It is trivial when a hard core is present as in LP. For superstable potentials this estimate is provided by Ruelle's result [6]. In the following lemma we extend this result to a Kac potential, proving the uniformity of the estimate for $\gamma \in(0,1]$.

Lemma 2.3. Let $v_{\gamma}$ satisfy D.1 and let there exist $\lambda>0$ (see D.3) such that D.4 and D.5 hold with the further condition

$$
A^{0}>2 \hat{\Phi}_{0} .
$$

Then for every regions $\Lambda$ and $\Delta$, bounded and Lebesgue measurable, there exist $\eta>0, \delta \geqq 0$, both independent of $\Lambda$ and $\gamma$, such that for every integer $m \geqq 0$ the probability that more than $m-1$ particles be in $\Delta$ is bounded from above as follows

$$
\begin{aligned}
Z^{-1}(\mu, \Lambda, \gamma) & \sum_{n=m}^{\infty} \sum_{p=0}^{\infty} \frac{\bar{\lambda}^{n+p} e^{\beta \mu(n+p)}}{n ! p !} \\
& \cdot \int_{(\Lambda \cap \Delta)^{n}} d x_{1} \ldots d x_{n} \int_{(\Lambda \backslash \Delta)^{p}} d x_{n+l} \ldots d x_{n+p} e^{-\beta U\left(x_{1} \ldots x_{n+p}\right)} \\
& <\exp \left[-\eta m^{2}+\delta m\right] .
\end{aligned}
$$

The proof of this lemma will be given in the Appendix. We only note here that the interest of the lemma lies in the fact that $\eta, \delta$ do not depend on $\gamma$.

We come now to the main point of the paper. For proving Eq. (1.3) we need an upper and a lower bound on the free energy density. The first one is provided by the corresponding estimate of LP which is in fact independent of the presence of hard cores. In the following theorem we will state an upper bound for the grand canonical pressure which will be later shown to lead to the required lower bound for the canonical free energy.

Theorem 1. Let $v_{\gamma}$ satisfy the hypotheses of Lemma 2.3 and let D.6 and D.7 hold; then

$$
\lim _{\gamma \rightarrow 0} \sup P(\mu, \gamma) \leqq \max _{\varrho}\left\{\mu \varrho-a^{0}(\varrho)-\frac{1}{2} \varrho^{2} \alpha\right\} .
$$

Proof. The main idea of the proof is to put a cut-off on the local density and to evaluate its effect through Lemma 2.3. Then the proof is analogous to that of the hard core case.

We suppose the region $\Lambda$ to be a cube of volume $(\lambda n L)^{v}$ where $L, n$ are positive odd integers. We consider $\Lambda$ divided into $L^{v}$ disjoint cubes of volume $(n \lambda)^{v}$. 
Then the asymptotic LP conditions are here fulfilled when $\lambda \ll n \ll \frac{1}{\gamma} \ll L$. This can be reproduced considering in the order the limits $L \rightarrow \infty, \frac{1}{\gamma} \rightarrow \infty, n \rightarrow \infty$.

We divide the configuration space into two disjoint regions, one in which no more than $M$ particles are present in each $\Gamma_{1}(r)$, and its complement. Then

$$
Z\left(\mu, \Gamma_{L n}(0), \gamma\right)=\sum_{m=0}^{\infty} \bar{\lambda}^{m} e^{\beta m \mu} Z_{c}^{\prime}\left(m, \Gamma_{L n}(0), \beta\right)+Z^{\prime \prime}
$$

where $Z_{c}^{\prime}$ is the function obtained from $Z\left(\mu, \Gamma_{L n}(0), \gamma\right)$ considering only the configurations in which no more than $M$ particles are in each $\Gamma_{1}(r)$ and $Z^{\prime \prime}$ is the contribution of the remaining configurations. From Lemma 2.2 we have

Then $\quad Z^{\prime \prime} \leqq\left[1-\left(1-e^{-\eta M^{2}+\delta M}\right)^{(L n)^{\nu}}\right] Z\left(\mu, \Gamma_{L n}(0), \gamma\right)$.

$$
\begin{gathered}
Z\left(\mu, \Gamma_{n L}(0), \gamma\right)\left(1-e^{-\eta M^{2}+\delta M}\right)^{(L n)^{v}} \\
\leqq \sum_{\substack{\left\{N_{r}\right\}=0 \\
|r| \leqq \frac{L-1}{2}}}^{M n^{v}}\left\{e^{\beta L^{v} D} e^{\beta \mu} \sum_{|i| \leqq \frac{L-1}{2}}^{\sum_{i}}\right. \\
\cdot e^{-\beta N_{(i, j)}^{\prime} W\left(i, j, N_{i}, N_{j}\right)} \prod_{|i| \leqq \frac{L-1}{2}} Z_{c}^{0}\left(N_{i}, \Gamma_{n}(0), \beta\right)
\end{gathered}
$$

where $N_{r}$ is the number of particles contained in $\Gamma_{n}(r)$. $\sum_{\left\{N_{r}\right\}=0,|r| \leqq \frac{L-1}{2}}^{M n^{v}}$ means sum on all the configurations in which no more than $M$ particles are present in each $\Gamma_{n}(r)$ with $|r| \leqq \frac{L-1}{2}$.

For the same configurations $D$ is a lower bound on the reference interaction of a cell $\Gamma_{n}(r)$ with all the others. From the lower regularity D.4

$$
D=-M^{2} \sum_{|r| \leqq \frac{n-1}{2}} \sum_{|s|>\frac{n-1}{2}} Q(|s-r|) .
$$

$W\left(i, j, N_{i}, N_{j}\right)$ is a lower bound on the interaction due to Kac potential between $N_{i}$ particles in $\Gamma_{n}(i)$ and $N_{j}$ in $\Gamma_{n}(j)$.

$\sum_{(i, j)}^{\prime}$ means sum on all the pairs of $i, j$ with $|i|,|j|<\frac{L-1}{2}$.

Following LP and remembering that in our assumptions the Kac potential is non positive

$$
\sum_{(i, j)}^{\prime} W\left(i, j, N_{i}, N_{j}\right) \geqq-\frac{1}{2} \sum_{|i| \leqq \frac{L-1}{2}} \frac{N_{i}^{2}}{(\lambda n)^{v}} c(\gamma)
$$


where

$$
c(\gamma)=\sum_{r \in \mathbb{Z}^{v}}\left|\inf _{\substack{x \in \Gamma_{n}(r) \\ y \in \Gamma_{n}(0)}} \gamma^{v} \varphi[\gamma(x-y)]\right|(\lambda n)^{\nu} .
$$

From Eqs. (2.18), (2.19) and (2.20) we have

$$
\begin{aligned}
P\left(\mu, \Gamma_{n L}(0), \gamma\right) \leqq-\frac{1}{\beta \lambda^{v}} \ln \left(1-e^{-\eta M^{2}+\delta M}\right) & \\
- & \frac{D}{(\lambda n)^{v}}+\frac{\ln \left[M(n L)^{v}\right]}{\beta(\lambda n L)^{v}} \\
& +\max _{N}\left\{\frac{\mu N}{(\lambda n)^{v}}+\frac{1}{2} \frac{N^{2}}{(\lambda n)^{2 v}} c(\gamma)-a_{c}^{0}\left(N, \Gamma_{n}, \beta\right)\right\}
\end{aligned}
$$

where we have bounded from above the terms in Eq. (2.17) by their maximum.

First we perform the limit $L \rightarrow \infty$ and so the third term of the r.h.s. of Eq. (2.21) vanishes.

Then we perform the limits $\gamma \rightarrow 0$ and $n \rightarrow \infty$. It is only the fourth term in Eq. (2.21) which requires some care. Let us study it. We introduce the free energy function $a_{c}^{0}\left(\varrho, \Gamma_{n}\right)$ also for values of $\varrho\left|\Gamma_{n}\right| \notin \mathbb{Z}^{+}$, by the usual linear interpolation. So that the maximum is not lowered by

$$
\max _{0 \leqq \varrho<\infty}\left\{\mu \varrho+\frac{1}{2} \varrho^{2} c(\gamma)-a_{c}^{0}\left(\varrho, \Gamma_{n}\right)\right\} .
$$

We now show that there exists $\varrho_{0}<\infty$ such that the maximum in Eq. (2.21) is reached in the interval $\left[0, \varrho_{0}\right]$ uniformly in $\gamma$ and $n$ satisfying $\gamma n \leqq 1$. In fact given $\varrho$ let $p$ the smallest integer greater than $\varrho(n \lambda)^{v}$.

$$
\begin{aligned}
& -a_{c}^{0}\left(\varrho, \Gamma_{n}(0), \beta\right) \leqq \frac{1}{\beta} \frac{1}{(n \lambda)^{v}} \ln \frac{\bar{\lambda}^{p}}{p !} \\
& \cdot \int_{\Gamma_{n}(0)} d x_{1} \ldots d x_{p} \exp \left\{-\beta \sum_{|r|<\frac{n-1}{2}}\left[A^{0} n^{2}(X, r)-B n(X, r)\right]\right\} \\
& \quad \leqq \frac{1}{\beta} \frac{1}{(n \lambda)^{v}} \ln \frac{\bar{\lambda}^{p}}{p !} \int_{\Gamma_{n}(0)} d x_{1} \ldots d x_{p} \exp (\beta B p) \exp \left(\frac{-\beta A^{0} p^{2}}{n^{v}}\right) \\
& \quad=\frac{1}{\beta} \frac{1}{(n \lambda)^{v}}\left\{\ln \left[\frac{\bar{\lambda}^{p}}{p !}(\lambda n)^{v} \exp (\beta B p)\right]-\frac{\beta A^{0} p^{2}}{n^{v}}\right\} \\
& \quad \leqq B\left(\varrho+\frac{1}{(n \lambda)^{v}}\right)+\frac{1}{\beta} \frac{1}{(n \lambda)^{v}} \ln \left[\frac{\bar{\lambda}^{p}}{p !}(\lambda n)^{v}\right]-\lambda^{v} A^{0} \varrho^{2} .
\end{aligned}
$$

We remember that

$$
c(\gamma) \leqq \lambda^{v} \hat{\Phi}_{\gamma n} \leqq \lambda^{v} A^{0}
$$

by D. 4 and because $\gamma n \leqq 1$. 
Then in Eq. (2.22) the leading term is $-\lambda^{v} A^{0} \varrho^{2}$ which for $\varrho \rightarrow \infty$ is infinitely negative. This proves the existence of $\varrho_{0}$ independent of $\gamma$ and $n$.

We now prove that $c(\gamma)$ has a limit when $\gamma \rightarrow 0,1 \geqq \gamma n \rightarrow 0$. The proof runs analogously to the one of Lemma 2.1 and we only give here an outline of it.

Let $\varphi(x)$ be continuous in $\mathscr{D} \subset \mathbb{R}^{v}$ so that $\mathbb{R}^{v}-\mathscr{D}$ is of zero Lebesgue measure. Then

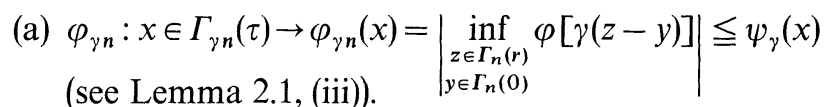

(b) $\int_{\substack{\mathbb{R}^{v} \\ \text { (see D.4). }}} d x \varphi_{\gamma n}(x)=\sum_{r \in \mathbb{Z}^{v}}(\gamma \lambda n)^{v} \inf _{\substack{x \in \Gamma_{n}(r) \\ y \in \Gamma_{n}(0)}} \varphi[\gamma(x-y)] \mid \leqq \lambda^{v} \hat{\Phi}_{\gamma n}$

(c) $\varphi_{\gamma n}(x) \rightarrow \varphi(x) \quad x \in \mathscr{D}$. $\gamma n \rightarrow 0$

(d) $\left|\varphi_{\gamma n}(x)\right| \leqq \psi(x)$.

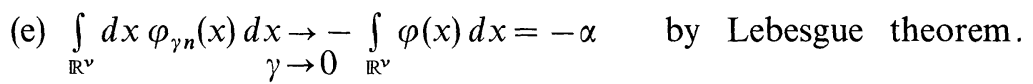

We finally note that $a^{0}\left(\varrho, \Gamma_{n}\right)$ is uniform in $\varrho$ when $n \rightarrow \infty$ in the interval $0 \leqq \varrho \leqq \varrho_{0}[7]$, and so is the term $\varrho^{2} c(\gamma)$ when $\gamma \rightarrow 0$. We can then interchange the maximum with the limits and we have

$$
\begin{gathered}
\lim _{\substack{\gamma \rightarrow 0 \\
n \rightarrow \infty \\
1 \geqq \gamma n \rightarrow 0}} \max _{0 \leqq \varrho \leqq \varrho_{0}}\left\{\mu \varrho+\frac{1}{2} \varrho^{2} c(\gamma)-a_{c}^{0}\left(\varrho, \Gamma_{n}\right)\right\} \\
=\max _{\varrho}\left\{\mu \varrho-\frac{1}{2} \varrho^{2} \alpha-a^{0}(\varrho)\right\} .
\end{gathered}
$$

Let us now consider the other terms in the r.h.s. of Eq. (2.21). In the same limit $\gamma n \rightarrow 0$ we have

In fact

$$
\lim _{n \rightarrow \infty} \frac{D}{n^{v}}=0
$$

$$
\begin{aligned}
\frac{|D|}{n^{v}} \leqq \text { const } \frac{M^{2}}{n^{v}} \sum_{|r| \leqq \frac{n-1}{2}} \sum_{|s|>\frac{n-1}{2}-|r|} Q(|s|) \\
=\frac{M^{2}}{n^{v}} \sum_{l=0}^{\frac{n-1}{2}}\left\{(2 l+1)^{v}-(2 l-1)^{v}\right\} \sum_{|s|>\frac{n-1}{2}-l} Q(|s|) .
\end{aligned}
$$

The leading term is

so Eq. (2.25) is proved.

$$
\frac{1}{n} \sum_{l^{\prime}=0}^{\frac{n-1}{2}} \sum_{|s|>l^{\prime}} Q(|s|) \rightarrow 0
$$


Finally we perform the limit $M \rightarrow \infty$. The first term of the r.h.s. of Eq. (2.21) vanishes and so Theorem 1 is proved.

Q.E.D.

Corollary. Let the hypothesis of Theorem 1 hold, then

$$
\liminf _{\gamma \rightarrow 0} a(\varrho, \gamma) \geqq C E\left\{a^{0}(\varrho)+\frac{1}{2} \varrho^{2} \alpha\right\} .
$$

Proof. We shall use the standard formula [8]

$$
a(\varrho, \gamma)=\max _{\mu}[\mu \varrho-P(\mu, \gamma)] \quad \gamma \in(0,1] .
$$

By Eq. (2.21) for every $\mu_{0} \in \mathbb{R}$

$$
\begin{aligned}
a(\varrho, \gamma) \geqq & \max _{\mu \leqq \mu_{0}}\left\{\mu \varrho-\max _{\varrho^{\prime}}\left[-\frac{1}{\beta \lambda^{v}} \ln \left(1-e^{-\eta M^{2}+\delta M}\right)\right.\right. \\
& \left.\left.-\frac{D}{(\lambda n)^{v}}+\mu \varrho^{\prime}+\frac{1}{2} \varrho^{\prime 2} c(\gamma)-a^{0} c\left(\varrho^{\prime}, \Gamma_{n}\right)\right]\right\} \\
= & \max _{\mu<\mu_{0}}\left\{\mu \varrho-\max _{\varrho^{\prime} \leqq \varrho_{0}}\left[-\frac{1}{\beta \lambda^{v}} \ln \left(1-e^{-\eta M^{2}+\delta M}\right)\right.\right. \\
& \left.\left.-\frac{D}{(\lambda n)^{v}}+\mu \varrho^{\prime}-C E\left\{a_{c}^{0}\left(\varrho^{\prime}, \Gamma_{n}\right)-\frac{1}{2} \varrho^{\prime 2} c(\gamma)\right\}\right]\right\}
\end{aligned}
$$

where $\varrho_{0}>\varrho$ has been introduced in Eq. (2.24).

We now use the following property proven in Ref. [4, I], Lemma 4. Let $0 \leqq \varrho<\varrho_{0}$, let $g(\varrho)$ be a convex function and let $A$ be greater than the slope of $g(\varrho)$ in $0 \leqq \varrho \leqq \varrho_{0}$ then

$$
\max _{\mu<A}\left[\mu \varrho-\max _{\varrho^{\prime}}\left(\mu \varrho^{\prime}-g\left(\varrho^{\prime}\right)\right]=g(\varrho) .\right.
$$

From Eq. (2.27) we then obtain

$$
\begin{aligned}
a(\varrho, \gamma) \geqq & -\frac{1}{\beta \lambda^{v}} \ln \left(1-e^{-\eta M^{2}+\delta M}\right)-\frac{D}{(\lambda n)^{v}} \\
& +C E\left\{a_{c}^{0}\left(\varrho, \Gamma_{n}\right)-\frac{1}{2} \varrho^{2} c(\gamma)\right\} .
\end{aligned}
$$

Performing the limits $\gamma \rightarrow 0, n \rightarrow \infty$ and $\gamma n \rightarrow 0$ and then $M \rightarrow \infty$ we finally get the thesis. We can interchange the limit $\gamma \rightarrow 0$ with the convex envelope because of the uniformity of the limit $\gamma \rightarrow 0$ in any interval of $\varrho$.

Theorem 2. Let $v_{\gamma}$ satisfy D.1 and D.6, let $\lambda>0$ exist (see D.3) such that D. 4 and D.5 hold and let $A^{0}>2 \hat{\Phi}_{0}$ then

$$
\lim _{\gamma \rightarrow 0} a(\varrho, \gamma)=C E\left\{a^{0}(\varrho)+\frac{1}{2} \alpha \varrho^{2}\right\} .
$$

Proof. The theorem follows from the previous corollary and from Eq. (2.25) of LP. 


\section{Appendix}

We discuss now the proof of Lemma 2.2. It follows strictly the corresponding one of Ruelle [6] Theorem 0.1. We use in this Appendix the same notation as in [6] and in particular the quantities $l_{j}, \alpha, V_{j}, P_{0},[q]$ are defined there.

The main change is in Proposition 2.1, which now reads:

Lemma A.1. Let $v_{\gamma}$ satisfy D.1 and let $\lambda>0$ (see D.3) exist such that D. 4 and D.5 hold with the further condition $A^{0}>2 \hat{\Phi}_{0}$. If $\alpha$ is sufficiently small one can choose $\sigma$ large enough and fix $P>P_{0}$ so that the following is true.

Let $n(\cdot)$ be a function from $\mathbb{Z}^{v}$ to the integers $\geqq 0$. Suppose that there exists $q$ such that $q \geqq P$ and $q$ is the largest integer for which

$$
\sum_{r \in[q]} n(r)^{2} \geqq \sigma V_{q} .
$$

Then exists $\zeta<1$ so that

$$
\begin{aligned}
& -\sum_{r \in[q+1]}\left[A n^{2}(r)-B n(r)\right]+\sum_{r \in[q+1]} \sum_{s \notin[q+1]}\{Q(|s-r|) \\
& \left.+\Phi_{\gamma}(|s-r|)\right\}\left\{\frac{1}{2} n(r)^{2}+\frac{1}{2} n(s)^{2}\right\} \leqq-(1-\zeta) A \sum_{r \in[q+1]} n^{2}(r) .
\end{aligned}
$$

Proof. We can prove the Lemma for $\gamma<\gamma_{0}$ where $\gamma_{0}>0$ is small enough. In fact for $\gamma>\gamma_{0}>0$ the hypothesis of Ref. [6] are fulfilled and the results of Ruelle apply directly.

We choose the constant $\sigma$ instead of the function $\psi(l)$ of Ref. [6]. Evidently its $\Psi(|r|)$ now is $Q(r)+\Phi_{\gamma}(|r|)$. Lemmas 2.2 and 2.3 of Ref. [6] hold. Lemma 2.4, which fixes the choice of $P$, now reads:

Lemma A.2. If $P>P_{0}$ is sufficiently large and $\gamma_{0}>0$ is sufficiently small, we have for $\gamma \in\left(0, \gamma_{0}\right]$ :

(a) $\sum_{s:|s|>l_{q+1}-l_{q}}\left[Q(|s|)+\Phi_{\gamma}(|s|)\right] \leqq \eta_{1} A$ with $\hat{\Phi}_{0} / A<\eta_{1}, q \geqq P$.

(b) $\sum_{k=1}^{\infty}\left(\Psi_{k}-\Psi_{k+1}\right) \sigma V_{q+k+2} \leqq \eta_{2} A \sigma \quad$ with $\quad \hat{\Phi}_{0} / A<\eta_{2}, q \geqq P$.

We prove (A.2). The contribution of the reference potential $Q$ to the r.h.s. of Eq. (A.2) can be made as small as we want by a convenient choice of $P$. The contribution of the Kac potential to the 1.h.s. can be bounded by $\hat{\Phi}_{\gamma}$, which for small $\gamma$ can be made as near as we want to $\hat{\Phi}_{0}$. So, given an $\eta_{1}>\frac{\hat{\Phi}_{0}}{A}$ we can choose $P_{1}, \gamma_{1}$ so that Eq. (A.2) holds for $\forall 0<\gamma<\gamma_{1}$, $P>P_{1}$. 
We prove Eq. (A.3). Again the contribution of the reference potential can be made as small as we want. For the Kac potential we must divide the sum in Eq. (A.3) into two parts:

$$
\begin{aligned}
& \sum_{k=1}^{N}\left\{\left[\Phi_{\gamma}\left(l_{q+k+1}-l_{q+1}+1\right)-\Phi_{\gamma}\left(l_{q+k+2}-l_{q+1}+1\right)\right] V_{q+k+2} \sigma\right\} \\
& +\sum_{k=N+1}^{\infty}\left\{\left[\Phi_{\gamma}\left(l_{q+k+1}-l_{q+1}+1\right)-\Phi_{\gamma}\left(l_{q+k+2}-l_{q+1}+1\right)\right] V_{q+k+2} \sigma\right\} .
\end{aligned}
$$

Using Lemma 2.3 (c) of Ref. [6] which states

$$
V_{q+k+2} \leqq\left(\frac{1+3 \alpha}{1-(1+\alpha)^{-k}}\right)^{v+1}\left(2 l_{q+k+1}-2 l_{q+1}+3\right)^{v}
$$

we have that Eq. (A.4) is

$$
\begin{aligned}
\leqq \sum_{k=1}^{N} \sigma\{ & {\left[\Phi_{\gamma}\left(l_{q+k+1}-l_{q+1}+1\right)-\Phi_{\gamma}\left(l_{q+k+2}-l_{q+1}+1\right)\right] } \\
\cdot & \left.\left(2 l_{q+k+1}-2 l_{q+1}+3\right)^{v}\right\}+\left(\frac{1+3 \alpha}{1-(1+\alpha)^{-k}}\right)^{v+1} \sum_{k=1}^{\infty} \sigma \\
\cdot & \left\{\left[\Phi_{\gamma}\left(l_{q+k+1}-l_{q+1}+1\right)-\Phi_{\gamma}\left(l_{q+k+2}-l_{q+1}+1\right)\right]\right. \\
\cdot & \left.\left(2 l_{q+k+1}-2 l_{q+1}+3\right)^{v}\right\} .
\end{aligned}
$$

We can choose $\alpha$ so small and then $N$ so large that

$$
\left(\frac{1+3 \alpha}{1-(1+\alpha)^{-N}}\right)^{v+1}<1+\varepsilon \quad \varepsilon \text { arbitrary small }
$$

Then when $\gamma \rightarrow 0$ the finite sum

$$
\sum_{k=1}^{N} \sigma\left\{\left[\Phi_{\gamma}\left(l_{q+k+1}-l_{q+1}+1\right)-\Phi_{\gamma}\left(l_{q+k+2}-l_{q+1}+1\right)\right]\left(2 l_{q+k+1}-2 l_{q+1}+3\right)^{v}\right\}
$$

vanishes and in Eq. (A.6)

$$
\sum_{k=1}^{\infty} \sigma\left\{\left[\Phi_{\gamma}\left(l_{q+k+1}-l_{q+1}+1\right)-\Phi_{\gamma}\left(l_{q+k+2}-l_{q+1}+1\right)\right]\left(2 l_{q+k+1}-2 l_{q+1}+3\right)^{\nu}\right\}
$$

tends to $\hat{\Phi}_{0}$. Therefore given $\eta_{2}>\hat{\Phi}_{0} / A, P_{2}$ and $\gamma_{2}$ can be found so that Eq. (A.3) is true for $\gamma \in\left(0, \gamma_{2}\right], P \geqq P_{2}$. Then Eqs. (A.2) and (A.3) hold at the same time for $P \geqq \max \left(P_{1}, P_{2}\right)$ and $\gamma \in\left(0, \gamma_{0}\right]$ where $\gamma_{0}=\min \left(\gamma_{1}, \gamma_{2}\right)$. 
We choose $\alpha, \gamma_{0}$ so that for $\gamma<\gamma_{0}$ the following are true

(i) $\left\{\left[\sum_{r} Q(|r|)\right]+\hat{\Phi}\right\}\left[(1+3 \alpha)^{2 v+2}-1\right]<\hat{\eta} A, \hat{\eta}>0$.

(ii) Lemma A.2 holds with $\eta_{1}, \eta_{2}>\hat{\Phi}_{0} / A$.

(iii) $\eta_{1}, \eta_{2}, \hat{\eta}$ are such that there exists $\zeta<1$ so that

$$
2 \zeta>\eta_{1}+\eta_{2}(1+3 \alpha)^{v+1}+\hat{\eta} .
$$

Then Lemma A.1 is proved by the same technique of Ref. [6], if we choose

$$
\sigma>\frac{B}{2 A}(1+3 \alpha)^{\nu+1}\left[2 \zeta-\eta_{1}-\eta_{2}(1+3 \alpha)^{\nu+1}-\hat{\eta}\right]^{-1} .
$$

The remaining steps of the proof of Lemma 2.2 are not substantially different from those of Ref. [6]; we have only to impose the further condition on $\sigma$ :

$$
\sigma>\frac{2(1+3 \alpha)^{v+1} \lambda^{\nu}\left(\bar{\lambda} e^{\beta \mu}\right)}{\beta(1-\zeta)}
$$

where $\bar{\lambda} e^{\beta \mu}$ is the activity.

\section{References}

1. Kac, M., Uhlenbeck, G. E., Hemmer, P.: Journ. Math. Phys. 4, 216 (1963).

2. van Kampen, N. G.: Phys. Rev. 135, A 362 (1964).

3. Lebowitz, J.L., Penrose, O.: Journ. Math. Phys. 7, 98 (1966).

4. Gates, D. J., Penrose, O.: Commun. math. Phys.15, 255 (1969); 16, 231 (1970); 17, 194 (1970).

5. Huang, K.: Statistical mechanics. New York: John Wiley \& Sons, Inc. 1963.

6. Ruelle, D.: Commun. math. Phys. 18, 127 (1970).

7. Ruelle,D.: Statistical mechanics, rigorous results. New York: Benjamin Inc. 1969.

8. Lebowitz, J. L.: Ann. Rev. Phys. Chem. 19, 389 (1968).
A. Gerardi
C. Marchioro
E. Olivieri

Istituto Matematico

Università di Roma

I-00100 Rome, Italy

\section{E. Presutti}

Istituto di Fisica

Università dell'Aquila

I-L'Aquila, Italy 
